\title{
Naming difficulties in alexia with agraphia for kanji after a left posterior inferior temporal lesion
}

\author{
Yasuhisa Sakurai, Katsuyuki Sakai, Manabu Sakuta, Makoto Iwata
}

\begin{abstract}
The case is described of a patient with alexia and agraphia for $k a n j i$, and severe anomia after a subcortical haemorrhage in the left posterior inferior temporal area. Magnetic resonance imaging at four months after onset showed a lesion in the inferior temporal and fusiform gyri, extending from the temporo-occipital junction toward the anterior third of the temporal lobe. Comparison with other reported cases of alexia with agraphia and anomia made it clear that when accompanied by severe anomia, the lesions extended either forward to the anterior part of the middle temporal gyrus or medially to the parahippocampal gyrus. It is suggested that the disconnection of association fibres between the parahippocampal, fusiform, middle, and inferior temporal gyri, especially between the parahippocampal gyrus and the other temporal gyri, or the cortical damage to the posterior part of these gyri is essential for the production of anomia.
\end{abstract}

(F Neurol Neurosurg Psychiatry 1994;57:609-613)

Since Iwata $^{1}$ first described a case of alexia with agraphia only for kanji (Japanese morphograms) due to a left posterior inferior temporal lesion, there have been many similar case reports stressing the importance of this area in the reading and writing of kanji. ${ }^{2-17}$ Alexia with agraphia for kanji due to a left posterior inferior temporal lesion has two main features. Firstly, it is often accompanied, to a greater or lesser degree, by naming difficulties (anomia). Little is known as to why anomia is caused by this lesion. Secondly, in some patients kana reading is more impaired than kanji reading, though kanji writing disturbance is always worse and persists longer than kana writing. For this reason it is called pure agraphia for kanji by some workers. ${ }^{215}$

In this paper we describe a Japanese man with alexia and agraphia for kanji accompanied by severe naming difficulties due to a left posterior inferior temporal lesion, and discuss the mechanism underlying the production of anomia by comparing this patient with reported cases.

\section{Case report}

Clinical details of the patient during the acute phase have been described elsewhere. ${ }^{17}$ The patient, a 71-year-old right-handed man, was educated to senior high school level. He had no history of serious illness. On 22 June 1991 he suddenly felt a slight left-sided headache. He then noticed that he could not remember the names of objects or his family and could not read newspapers or write anything but his own name. He was admitted to the Japanese Red Cross Medical Center on 22 June with a diagnosis of cerebrovascular disease.

He was fully conscious on admission. Cranial nerve function, motor system, cutaneous and deep sensation, and coordination were all intact except for a mild hearing loss in both ears. There was no visual field defect. Blood chemistry was normal. Audiometry showed mild hearing loss in both ears for speech discrimination (right ear, $75 \%$; left ear, $80 \%$; 80-85 dB).

\section{NEUROPSYCHOLOGICAL EXAMINATION}

The patient's verbal IQ with the Wechsler adult intelligence scale-revised (WAIS-R) was 80 , and his performance IQ was 86 .

The Western aphasia battery (WAB; Japanese edition $^{18}$ (table 1)) at two months showed that his spontaneous speech was fluent with preserved prosody. He showed marked word finding difficulty and overused demonstrative pronouns, but there were few paraphasias. He made three correct responses out of 20 in object naming and, given phonemic cues, made another four correct responses. Circumlocution was observed in most of the incorrect responses. $\mathrm{He}$ could explain how to use objects and could point to

Table 1 Scores for the Western aphasia battery

\begin{tabular}{lll}
\hline & August 1991 & October 1992 \\
\hline Spontaneous speech & & \\
Information content & $7 / 10$ & $9 / 10$ \\
Fluency & $8 / 10$ & $9 / 10$ \\
Naming total & $2 \cdot 4 / 10$ & $5 \cdot 7 / 10$ \\
Object naming & $13 / 60$ & $31 / 60$ \\
Repetition & $8 \cdot 6 / 10$ & $9 \cdot 6 / 10$ \\
Comprehension total & $7 / 10$ & $9 \cdot 45 / 10$ \\
Yes/no questions & $51 / 60$ & $54 / 60$ \\
Auditory word recognition & $41 / 60$ & $59 / 60$ \\
Sequential commands & $48 / 80$ & $76 / 80$ \\
Reading total & $3 \cdot 9 / 10$ & $8 \cdot 05 / 10$ \\
Comprehension of sentences & $4 / 40$ & $40 / 40$ \\
Reading commands & $7 \cdot 5 / 20$ & $13 / 20$ \\
Kanji to object matching & $3 / 3$ & $3 / 3$ \\
Kana to object matching & $3 / 3$ & $3 / 3$ \\
Kanji to picture matching & $3 / 3$ & $3 / 3$ \\
Kana to picture matching & $3 / 3$ & $3 / 3$ \\
Writing total & $6 \cdot 55 / 10$ & $7 \cdot 5 / 10$ \\
Copying & $10 / 10$ & $10 / 10$ \\
Kanji writing to dictation & $0 / 6$ & $2 / 6$ \\
Kana writing to dictation & $4 / 6$ & $5 / 6$ \\
Sentence writing to dictation & $7 / 10$ & $9 / 10$ \\
Praxis total & $9 \cdot 8 / 10$ & $9 \cdot 8 / 10$ \\
Construction total & $8 / 10$ & $7 \cdot 9 / 10$ \\
\hline
\end{tabular}


objects on command fairly well (17/20). Repetition was almost normal. Auditory comprehension was slightly impaired. Kanji reading was poor, which resulted in a low score for comprehension of written language, whereas kana reading was generally good, though with a few phonemic paraphasias (this difference was made clear in the reading of sentences including both kanji and kana, but was not reflected in the WAB score). In kanji-to-object matching he could point to the appropriate objects correctly, though he could only read one of six kanji word items. This implies that semantics were preserved even when he could not read kanji words, characteristic of deep dyslexia. ${ }^{19}$ Also, in writing to dictation, kanji was inferior to kana. Agraphia of kanji due to graphias of kana were noted. The patient's

Figure 1 CT scan on admission showing a large high density area in the left temporal subcortical structure extending to the anterior part of the temporal lobe. The left is the patient's left side. word recall difficulty and phonemic para-

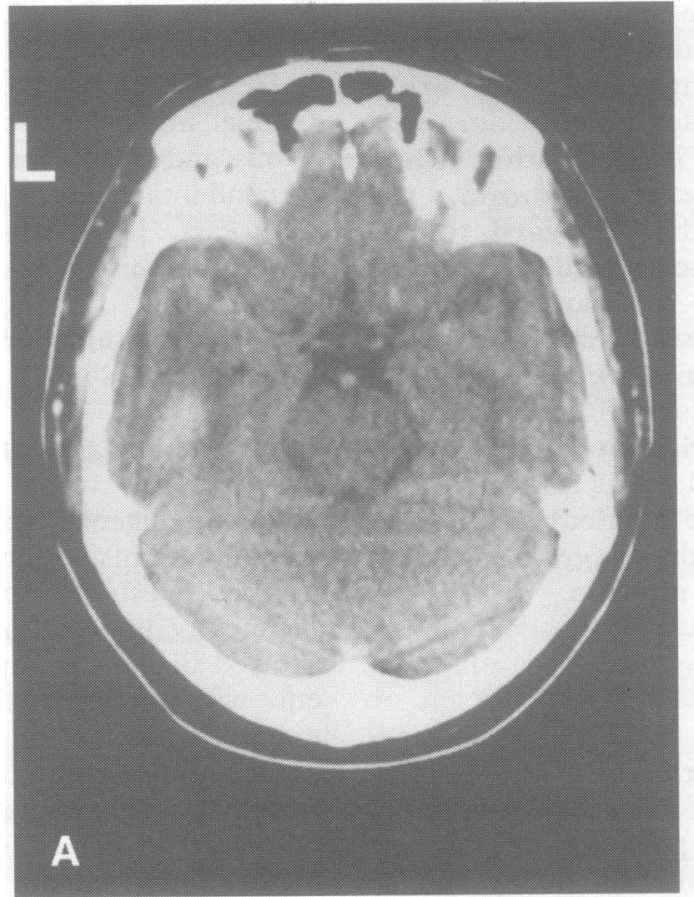

speech profile was rated as one of anomic aphasia and alexia with agraphia, relatively selective for kanji. He did not show apraxia, a construction disturbance, nor colour agnosia.

To evaluate the extent of the reading and writing difficulties for kanji compared with kana more precisely, at three months the patient was given reading aloud and writing to dictation tasks involving 100 kanji and the corresponding kana (readings of kanji), all of which are taught in the first three years of primary school. The results showed that in both reading (correct response in kanji, 20\%; kana, $75 \% ; \mathrm{p}<0.001$ on the two-tailed Fisher's exact method) and writing to dictation (correct response in kanji, 4\%; kana, 59\%; p < 0.001 on the two-tailed Fisher's exact method), kana function was significantly superior to kanji function. Most of the errors
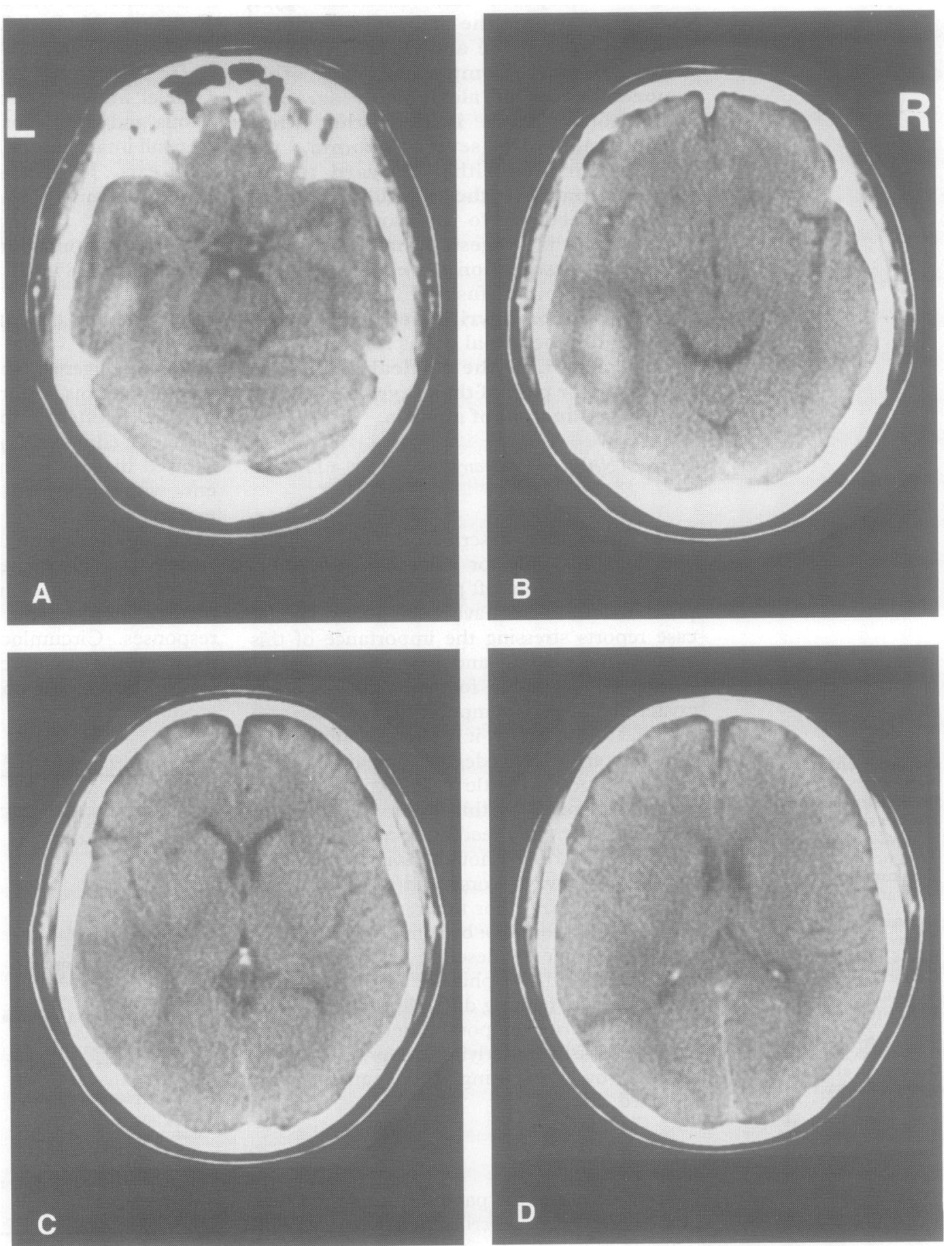
comprised no response in kanji tasks and phonemic paralexias or paragraphias in kana tasks. A similar tendency was observed on re-examination 15 months after onset with significant improvement (reading: correct response in kanji, 46\%, $\mathrm{p}<0.005$; kana, $91 \%, \mathrm{p}<0.05$; writing to dictation: correct response in kanji, $12 \%, \mathrm{p}<0.005$; kana, $77 \%, \mathrm{p}<0.005$, on the McNemar test). On the whole, he could read in kana more than $90 \%$ of the items he read correctly in kanji, as well as in writing. He could also read almost

Figure 2 MRI T2weighted coronal view $(0.5$ $T ; T R=2500 \mathrm{~ms} ; T E=$ $120 \mathrm{~ms}$ ) through the atrium of the lateral ventricle $(A)$ and hippocampus $(B, C)$ at four months. The right is the patient's left side. $A$ low intensity area suggesting haemosiderin deposition is present in the cortico-subcortical tissue of the left fusiform, inferior temporal gyri from the occipital to the anterior third of the temporal lobe.
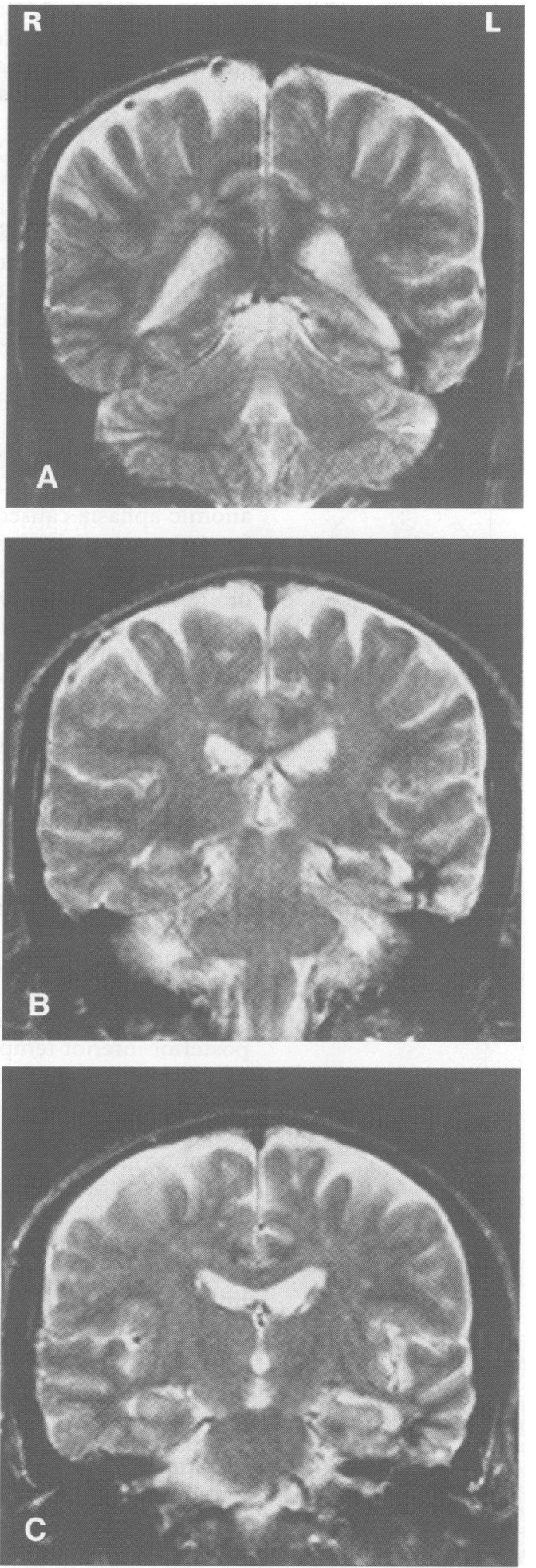

all kanji or kana he could write in the reexamination, though there were some kanji or kana which he could not read but could write, or vice versa, in the first test (number of items with both correct reading and correct writing were two for kanji and 45 for kana).

Severe naming difficulties and alexia with agraphia for kanji still persisted at 16 months, though there was some improvement (table 1).

CT on admission showed a high density area in the left temporal subcortical structure, thus the patient was diagnosed as having a cerebral haemorrhage (fig 1). There was also a low density area suggesting an old infarction in the anterolateral portion of the left putamen. MRI at the same time confirmed these lesions and there was no other change in intensity affecting the left thalamus. In MRI at four months the lesion was evident in the cortico-subcortical tissue of the left inferior temporal and fusiform gyri, probably affecting the inferior longitudinal fasciculus and extending from the temporo-occipital junction toward the anterior third of the temporal lobe (fig 2). Single photon emission CT with iodoamphetamine labelled with iodine-123 (123I-IMP-SPECT) at four months showed decreased blood flow in the lower and medial parts of the left temporal lobe, including the hippocampal region, whereas the regional cerebral blood flow in the left basal ganglia and thalamus was preserved.

\section{Discussion}

The patient presented with alexia with agraphia for kanji and severe naming difficulties after a subcortical haemorrhage of the left posterior inferior temporal area. Anomia can also be observed in a thalamic lesion. ${ }^{20}$ The haematoma and its surrounding oedema in the acute phase could have some effect on the left thalamus, but MRI at this time showed that there was no change in intensity in the thalamus. In addition, the patient's severe anomia was still present at 12 months. Therefore, we cannot attribute anomia to the acute dysfunction of the thalamus.

The patient had an old infarction in the anterolateral portion of the left putamen. A basal ganglia lesion can also produce atypical aphasia. ${ }^{21}$ In such a case, however, the lesion affects more widespread areas including the head of the caudate nucleus, the putamen, and the internal capsule. The patient's putaminal lesion was too small to cause aphasia, and the regional cerebral blood flow of the left basal ganglia was preserved. Furthermore, the patient did not show anomia until admission, thus, the old infarction in the putamen cannot cause the anomia.

There have been more than 20 case reports of alexia with agraphia due to a left posterior inferior temporal lesion. Severe naming difficulties often occurred in those patients in whom kanji reading was more impaired, whereas in patients with more impaired kana reading naming difficulties were, if present, only slight. Thus to correlate the lesion with 
Table 2 Classification of alexia with agraphia for kanji with significant difference in reading between kanji and kana. Values are mean (\%) scores

\begin{tabular}{|c|c|c|c|c|}
\hline \multirow[b]{2}{*}{ Patient group ${ }^{*}$} & \multirow[b]{2}{*}{ Naming } & \multicolumn{2}{|l|}{ Reading } & \multirow[b]{2}{*}{ Lesion† } \\
\hline & & kana & kanji & \\
\hline \multicolumn{5}{|l|}{ Group A } \\
\hline $\begin{array}{l}\text { Shiota et al }{ }^{3} \\
\text { Kawamura }^{5}\end{array}$ & sl. & $20 / 36(56)$ & $20 / 24(83)$ & PITsł \\
\hline $\begin{array}{l}\text { Patient TN } \\
\text { Mochizuki and Ohtomo }\end{array}$ & sl. & $\begin{array}{l}263 / 416(63) \\
8 / 48(17)\end{array}$ & $\begin{array}{l}402 / 416(92) \\
74 / 100(74)\end{array}$ & $\begin{array}{l}\text { Fu, inf } T, T-O \ddagger \ddagger \\
\text { Inf } T, O \neq \ddagger\end{array}$ \\
\hline \multicolumn{5}{|l|}{ Group B } \\
\hline $\begin{array}{l}\text { Oka et al }{ }^{9} \\
\text { Kawahata et al }{ }^{10}\end{array}$ & $\begin{array}{l}16 / 20(80 \%) \\
18 / 20(90 \%)\end{array}$ & $\begin{array}{l}752 / 800(94) \\
24 / 25(96)\end{array}$ & $\begin{array}{l}674 / 800(84) \\
2 / 25(8)\end{array}$ & 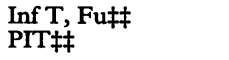 \\
\hline \multicolumn{5}{|l|}{ Group C } \\
\hline $\begin{array}{l}\text { Imamura et al }{ }^{12} \\
\text { Notoya et al }{ }^{13} \\
\text { Soma et al }{ }^{15} \\
\text { Patient } 2\end{array}$ & $\begin{array}{c}0 / 20(0 \%) \\
11 / 20(55 \%) \\
33 / 50(66 \%)\end{array}$ & $\begin{array}{l}28 / 30(93) \\
20 / 20(100) \\
219 / 221(99)\end{array}$ & $\begin{array}{l}9 / 30(30) \\
12 / 20(60) \\
201 / 221(91)\end{array}$ & 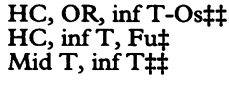 \\
\hline $\begin{array}{l}\text { Notoya et al }{ }^{16} \\
\text { This patient }\end{array}$ & $\begin{array}{l}7 / 20(35 \%) \\
3 / 20(15 \%)\end{array}$ & $\begin{array}{l}20 / 20(100) \\
75 / 100(75)\end{array}$ & $\begin{array}{l}14 / 20(70) \\
20 / 100(20)\end{array}$ & $\begin{array}{l}\text { Mid T, inf T, T-O } \\
\text { Inf T, Fuł }\end{array}$ \\
\hline
\end{tabular}

${ }^{\star}$ Group A, more impaired kana reading; group $\mathrm{B}$, more impaired kanji reading with slight or $\mathrm{n}$ anomia; and group $C$, more impaired kanji reading with profound anomia. +Based on the authors' evaluation. $\neq \mathrm{p}<0.05$, $\neq \neq \mathrm{p}<0.01$ on the two-tailed Fisher's exact method between $k a n j$ reading and kana reading. Abbreviations: PIT $=$ posterior inferior temporal; inf $=$ inferior sl. = slightly disturbed; $N=$ normal; $T=$ temporal; $O=$ occipital; $s=$ subcortical; $F u=$ fusiform; $\mathrm{HC}=$ hippocampus; $\mathrm{OR}=$ optic radiation .

the symptom we divided the reported cases of alexia with agraphia due to a posterior inferior temporal lesion into three groups: group A, patients with more impaired kana reading ${ }^{3-7}$; group B, patients with more impaired kanji reading and slight or no anomia $(80 \%$ or more correct in confrontation naming $)^{-11}$; and group $\mathrm{C}$, patients with more impaired kanji

$\mathbf{L}$

A
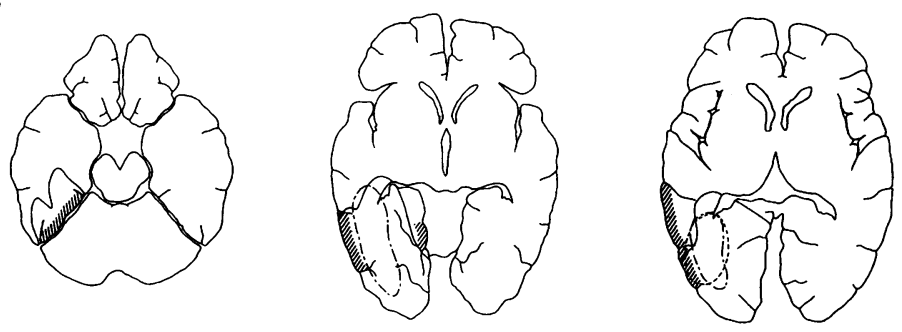

B
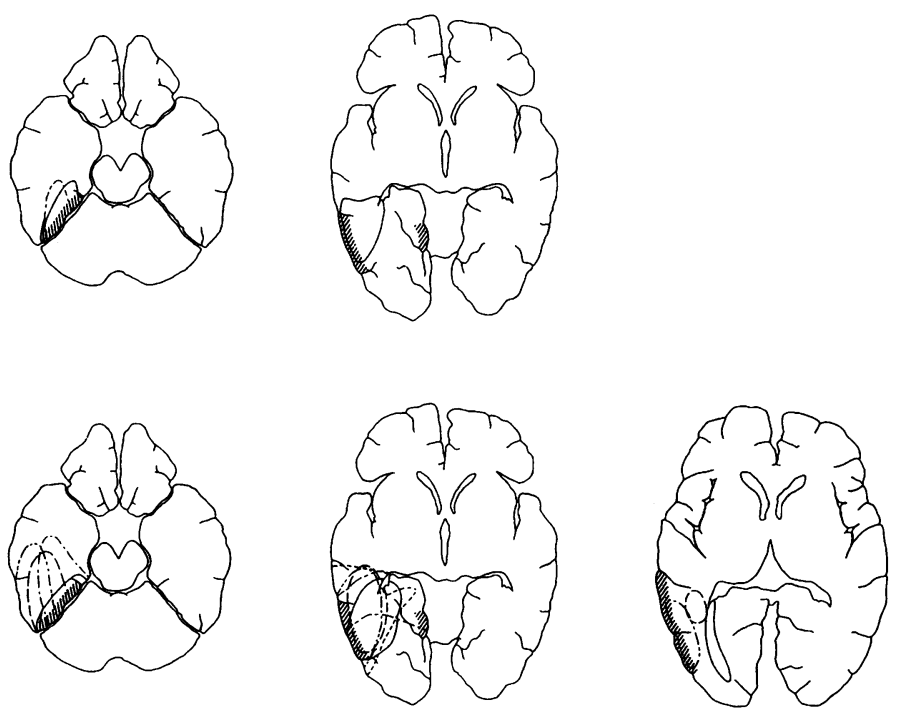

Figure 3 Superimposed schematic CT images of alexia with agraphia due to a left posterior inferior temporal lesion. The left is the patients' left side. Areas with oblique lines denote the left posterior inferior temporal area (almost corresponding to Brodmann's area 37). (A) Patients with significantly more impaired kana reading. ${ }^{35} 6$ (B) Patients with significantly more impaired kanji reading and slight or no anomia. ${ }^{910}$ (C) Patients with significantly more impaired kanji reading and severe anomia, including our patient. ${ }^{12}{ }^{13}{ }^{15-17}$ The lesion in alexia with agraphia for kanji is usually localised in the left posterior inferior temporal area $(B)$. When kana reading impairment is more pronounced, the lesion affects the lateral occipital area $(A)$. If it accompanies severe anomia, the lesion extends forward to the anterior part of the temporal lobe or medially to the parahippocampal gyrus (C). reading and severe anomia (less than $80 \%$ correct in confrontation naming). ${ }^{12-17}$

We determined which was more impaired, $k a n j i$ or kana, in each patient based on the scores for the individual kanji and kana reading test and the authors' evaluation. We then chose patients with significantly more impaired kana or kanji reading from each group ( $p<0.05$ or less on the Fisher's exact method; table 2) and traced their lesion on CT axial slices. Figure 3 shows the superimposed lesion images of each group. We can see from this figure that in group A patients the lesion extends from the left posterior inferior temporal area backward to the lateral occipital gyrus. In alexia with agraphia for kanji without profound anomia (group B) the lesion is mostly localised in the left posterior inferior temporal area (almost corresponding to Brodmann area 37). When severe anomia accompanies alexia with agraphia for kanji (group C), the lesion affects the left posterior inferior temporal area, extending either forward as far as the anterior part of the middle temporal gyrus or medially to the parahippocampal gyrus. As this inference is based on a small number of patients, we need further evidence to confirm it.

This patient showed severe anomia, but he could explain how to use objects and point to objects almost always correctly on command.

$\boldsymbol{R}$ Such a symptom is characteristic of pure anomic aphasia caused by a localised lesion. ${ }^{22}$ In contrast, a patient with anomia with a cortical degenerative disease such as Pick's disease or Mesulam's slowly progressive aphasia ${ }^{23}$ cannot explain how to use objects, recognise them, or point to objects on command. ${ }^{24}$ As this patient did not improve with tactile cues and could copy and match figures to sample, the naming disturbance was not caused by visual agnosia. This difference is probably because in anomia caused by a localised lesion, lexical information is still preserved in the cortex but its use is impaired, whereas in anomia observed in a cortical degenerative disease lexico-semantic information itself is affected.

Comparison of the lesions of group B patients with those of group $C$ patients showed that the localised damage to the left posterior inferior temporal area (posterior part of the middle and inferior temporal area and fusiform gyri) did not produce anomia and that severe anomia occurred only when the posterior inferior temporal lesion extended forward to the anterior part of the temporal lobe or medially to the parahippocampal gyrus. We identified the extent of the lesion in our patient in more detail on MRI coronal images. It affected the point at which fibres from the fusiform, middle, and inferior temporal gyri seemed to converge, extending from the temporo-occipital junction to the anterior third of the temporal lobe. It was continuous along almost the whole length of the parahippocampal gyrus as a result, separating the parahippocampal gyrus from the other temporal gyri (fig 2). A similar lesion was reported in a patient with severe anomia and alexia with 
agraphia for kanji due to cerebral infarction. ${ }^{14}$ These findings suggest that anomia occurs when the association fibres are disconnected between the parahippocampal, fusiform, middle, and inferior temporal gyri, or when the lesion affects the parahippocampal gyrus. The patient of Mochizuki and Ohtomo ${ }^{6}$ (group A, delineated by solid lines in fig 3A) does not seem to fit our view in that there was no anomia though the lesion extended forward in the temporal lobe. In this patient, however, the damage was, according to the MRI finding, restricted to the occipital lobe and the inferior temporal gyrus, whereas the lingual, fusiform, parahippocampal, superior and middle temporal gyri were spared. This patient shows that anomia does not appear if the posterior part of the parahippocampal gyrus is intact.

In our patient the parahippocampal gyrus and hippocampus were apparently spared, but SPECT showed the decreased blood flow in the left inferior and medial temporal area, including the hippocampal region. This implies that the lesion had some effect on the hippocampal memory system.

In monkeys the parahippocampal gyrus and perirhinal cortex have afferent and efferent connections with several association areas in the frontal, temporal, and parietal lobes. ${ }^{25}$ Given these findings, it is assumed that the disconnection of association fibres between the parahippocampal, fusiform, middle, and inferior temporal gyri, especially between the parahippocampal gyrus and the other temporal gyri, or the cortical damage to the posterior part of these gyri is essential for the production of pure anomic aphasia.

We found in a review of published work $^{17}$ that the more severe the naming difficulties were, the more impaired kanji writing seemed to be, and this was dependent on the extent of the lesion. The combination of anomia and alexia with agraphia for kanji is observed only in Japan. The same lesion may result in anomia and reading and writing difficulties of irregular words in English-speaking countries.

We are grateful to Professor Ichiro Kanazawa, Department of Neurology, University of Tokyo School of Medicine, for his Neurology, University of Tokyo School of Medicine, for his
support and encouragement. We also express our thanks to Dr support and encouragement. We also express our thanks to Dr CT films and to Dr Hiroshi Mochizuki, Tohoku University School of Medicine for informing us of his case in detail.

1 Iwata M. Kanji versus Kana. Neuropsychological correlates of the Japanese writing system. Trends Neurosci 1984;7:290-3.

2 Yokota $\mathrm{T}$, Ishiai S, Furukawa $\mathrm{T}$, Tsukagoshi $\mathrm{H}$. Pure agraphia of kanji due to thrombosis of the Labbé vein. $\mathcal{F}$
Neurol Neurosurg Psychiatry 1990;53:335-8.

3 Shiota J, Kawamura M, Isono O, Hirayama K. Alexia with agraphia produced by a localized infarction in the inferior posterior region of the left temporal lobe [in Japanese]. No to Shinkei 1986;38:1051-5.

4 Shimomura T, Tagawa K, Nagata K, Shishido F. A case of alexia with agraphia due to the left temporo-occipital lobar hematoma [in Japanese]. Shinkeinaika 1987;26: 57-64.

5 Kawamura M. Non-classical type alexia without agraphia [in Japanese]. Shitsugosho Kenkyu 1988;8:185-93.

6 Mochizuki H, Ohtomo R. Pure alexia in Japanese and agraphia without alexia in kanji. The ability dissociation between reading and writing in kanji vs kana. Arch Neurol 1988;45:1157-9.

7 Shimomura T, Suzuki T, Takahashi S. Two cases of alexia with agraphia due to the left posterior inferior alexia with agraphia due to the left posterior inferior temporal

8 Iwata $M$. Neural mechanisms of reading and writing. Neurogrammatological approach. In: Tsukada Y, ed. Perspectives on neuroscience. From molecule to mind. Tokyo: University of Tokyo Press, 1985:299-312.

9 Oka H, Asano T, Hattori S, Mochio S, Sugishita M. Pure alexia and alexia with agraphia caused by lesions of the left inferior temporal and fusiform gyri [in Japanese]. Shinkeinaika 1985;23:73-6.

10 Kawahata N, Tagawa K, Hirata Y, Nagata K, Shishido F. A case of alexia with agraphia due to the infarction in the left inferior-posterior temporal lobe [in Japanese]. Rinshoshinkeigaku 1987;27:420-7.

11 Kawamura M, Hirayama $K$, Hasegawa $K$, Takahashi N, Yamamura A. Alexia with agraphia of kanji Japanese Yamamura A. Alexia with agraphia of kanji Japanese morphog

12 Imamura S, Ohno N, Nakashima A, Yamanaga H, Ideta $\mathrm{T}$. Alexia with agraphia caused by temporo-occipital infarcts of the dominant side. Case report [in Japanese]. Shinkeinaika 1985;23:362-8.

13 Notoya M, Suzuki S, Kurachi M, Koyama Y, Hiramatsu $H$. A case of alexia with agraphia caused by a left inferior temporal lesion [in Japanese]. Shinkeishinrigaku 1987; 3:244-50.

14 Kaneki $M$, Takeda $K$, Bandoh $M$, Iwata $M$, Mannen $T$, Sekiguchi S. A case of amnesic aphasia and alexia with agraphia caused by a subcortical infarction of the left inferior temporal area [in Japanese] [abstract]. Shinkeishinrigaku 1988;4:33.

15 Soma Y, Sugishita M, Kitamura K, Maruyama S, Imanaga $H$. Lexical agraphia in the Japanese language. Pure agraphia for kanji due to left posteroinferior temporal agraphia for kanji due to left poste
lesions. Brain 1989;112:1549-61.

16 Notoya $M$, Tetoriya H, Suzuki S, Fujii H, Furukawa $H$. Alexia with agraphia of kanji of the left inferior temporal gyrus type [in Japanese]. Shitsugosho Kenkyu 1991;11: $140-6$.

17 Sakai K, Sakurai Y, Sakuta M, Iwata M. Naming difficulties seen in a case of alexia with agraphia caused by a left postero-inferior temporal lesion [in Japanese]. Rinshoshinkeigaku 1992;32:1227-31.

18 Kertesz A. The Western aphasia battery. New York: Grune and Stratton, 1982 (translated into Japanese: Sugishita $M$, et al. Tokyo: Igaku-Shoin, 1986).

19 Newcombe F, Marshall JC. On psycholinguistic classifications of the acquired dyslexias. Bull Orton Soc 1981; 31:29-46.

20 Graff-Radford NR, Eslinger PJ, Damasio AR, Yamada T. Nonhemorrhagic infarction of the thalamus: behavioral, anatomic, and physiologic correlates. Neurology 1984;34: 14-23.

21 Damasio AR, Damasio H, Rizzo M, Varney N, Gersh F. Aphasia with nonhemorrhagic lesions in the basal ganglia and internal capsule. Arch Neurol 1982;39: $15-20$

22 Benson BF. Neurologic correlates of anomia. In: Whitaker H, Whitaker HA, eds. Studies in neurolinguistics. Vol. 4. New York: Academic Press, 1979:293-328.

23 Mesulam M-M. Slowly progressive aphasia without generalized dementia. Ann Neurol 1982;11:592-8.

24 Sakurai Y, Momose T, Watanabe T, Bandoh M, Ishikawa $T$, Iwata $M$. Slowly progressive fluent aphasia. Clinical features and an imaging study including MRI, SPECT and PET [in Japanese]. Rinshoshinkeigaku 1992;31: 505-11.

25 Squire LR, Zola-Morgan S. Memory: brain systems and behavior. Trends Neurosci 1988;11:170-5. 